\title{
Preoperative muscle thickness influences muscle activation after arthroscopic knee surgery
}

\author{
Jorge Amestoy ${ }^{1,2}$. Daniel Pérez-Prieto ${ }^{1,2}$ (]) $\cdot$ Raúl Torres-Claramunt ${ }^{1,2} \cdot$ Juan Francisco Sánchez-Soler ${ }^{1,2}$. \\ Albert Solano $^{1,2} \cdot$ Joan Leal-Blanquet ${ }^{1,2} \cdot$ Pedro Hinarejos $^{1,2} \cdot$ Joan Carles Monllau $^{1,2}$
}

Received: 26 July 2021 / Accepted: 25 November 2021 / Published online: 18 December 2021

(c) The Author(s) 2021

\begin{abstract}
Purpose The aim of this study was to compare the correlation between preoperative quadriceps femoris muscle thickness and postoperative neuromuscular activation and quadriceps femoris strength in patients with and without patellofemoral pain after arthroscopic partial meniscectomy.

Methods A series of 120 patients were prospectively analysed in a longitudinal cohort study of patients scheduled for arthroscopic partial meniscectomy. The patellofemoral pain group included patients who developed anterior knee pain after surgery while the control group included those who had not done so. Patients with preoperative patellofemoral pain, previous knee surgeries as well as those on whom additional surgical procedures had been performed were excluded. Of the 120 initially included in the study, 90 patients were analysed after the exclusions.

Results There is a direct correlation between preoperative quadriceps femoris muscle thickness and the neuromuscular activity values and the strength of the muscle at 6 weeks after surgery. These results were seen exclusively in the group of patients who do not develop patellofemoral pain $(0.543, p=0.008)$. The group of patients who developed anterior knee pain in the postoperative period did not show this correlation (n.s.).

Conclusion In patients without patellofemoral pain after meniscectomy, the greater the preoperative thickness of the quadriceps femoris, the more postoperative neuromuscular activation and strength they had. This correlation did not occur in those patients who develop patellofemoral pain after meniscal surgery.
\end{abstract}

Level of evidence II.

Keywords Knee arthroscopy · Quadriceps muscle activation · Quadriceps muscle atrophy · Patellofemoral pain · Quadriceps muscle strength

\section{Introduction}

Quadriceps activation failure (QAF) occurs due to alterations in neural signalling caused by a reduction in alpha motor neuron pool recruitment and/or firing rate [12]. It commonly occurs after knee surgery and is not simply an isolated local phenomenon related to atrophy. This has been attributed to arthrogenic muscle inhibition, [21] a process in which quadriceps activation failure is caused by neural inhibition [30].
Daniel Pérez-Prieto
dr.danielperezprieto@gmail.com
1 Hospital del Mar, Barcelona, Spain
2 Autonomous University of Barcelona, Barcelona, Spain

Activation failure is the inability to completely volitionally contract the muscle due to alterations in neural signalling. It is common following any type of knee surgery [12, 14]. If left untreated, QAF can significantly impede strength gains by only allowing portions of the muscles to be volitionally utilized during active exercise [13, 19].

If these neural abnormalities are not targeted with specific interventions used to disinhibit an inhibited muscle, quadriceps dysfunction may persist and become a factor limiting successful postoperative knee management $[17,19]$.

Quadriceps muscle hypotrophy (QMH) that occurs following knee surgery is also thought to contribute to persistent muscle weakness $[17,39]$ due to alterations in muscle architecture [23], selective fibre atrophy [20, 21], or even neural deficits like QAF [25]. It might cause patellofemoral pain (PFP), a dreaded complication after knee surgery. It 
affects up to $23 \%$ of patients who undergo arthroscopic partial meniscectomy (APM) [1, 5].

Muscle hypotrophy as well as the delayed onset of electromyographic activity of the quadriceps femoris muscle after arthroscopic partial meniscectomy predispose to the development of postoperative PFP. Furthermore, these two risk factors also predispose to worse postoperative functional results [1].

Despite the important role that the quadriceps muscle plays in this pathology, whether having greater quadriceps muscle thickness before surgery has any impact in the neuromuscular activation of this muscle in the postoperative period has not yet been studied. No prospective study investigating the development of patellofemoral pain after a knee arthroscopy has tested the electromyographic activity of the vastus medialis and vastus lateralis muscles and its relationship with the preoperative muscular thickness.

The aim of this study was to compare the correlation between preoperative quadriceps muscle thickness, its postoperative neuromuscular activation and strength in patients with and without patellofemoral pain after APM.

The hypothesis was that there is direct correlation between the preoperative quadriceps muscle thickness and its neuromuscular activity after an APM in patients who do not develop patellofemoral pain.

\section{Materials and methods}

Approval for the study was granted by the Ethics Committee of Clinical Research of Parc de Salut Mar Hospital, Autonomous University of Barcelona (CEIC no. 2014/5534). Between 2015 and 2017, a prospective longitudinal cohort study was carried out on consecutive patients who were scheduled to undergo APM. The inclusion criteria were that the patient be aged 18 years or older and have an acute symptomatic medial meniscal tear requiring surgery. All patients underwent the procedure at a maximum of 6 months of evolution from the meniscal tear. No differences were found in the time of evolution of the meniscal tear between the groups. The exclusion criteria included having had PFP prior to surgery, previous surgeries on the involved knee (including meniscal repair) or if there had been an associated surgical procedure (e.g. chondral repair, ACL reconstruction, etc.) during the index procedure.

For the reasons previously stated, 30 patients out of the 120 initially included in the study were excluded. Nineteen of the 30 had had PFP before surgery. The remaining 11 patients of those 30 had undergone an associated surgical procedure like meniscal repair (7), microfractures due to the incidental presence of a chondral injury (3), and there was 1 partial meniscal injury that was left untreated (Fig. 1).

\section{Surgical procedure}

The same team of knee surgeons from Parc de Salut Mar Hospital carried out all the surgical procedures on the
Fig. 1 Flowchart of the study and enrolment of the patients. PFP, patellofemoral pain

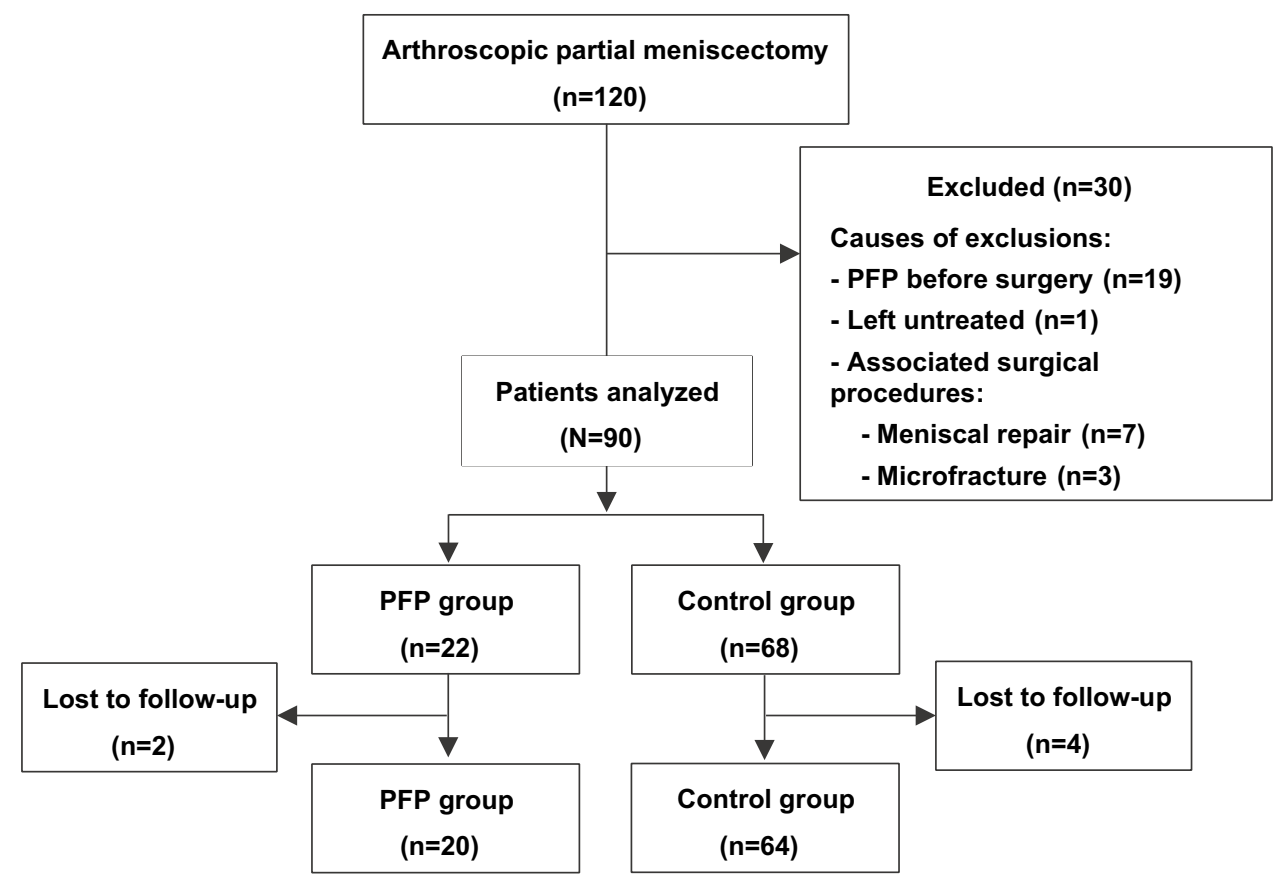


patients. They were done with the patients under spinal anaesthesia (15 mg levobupivacaine $0.5 \%$ ). As the surgery was of short duration, a tourniquet was used at a pressure of $100 \mathrm{mmHg}$ above systolic pressure with prior exsanguination of the limb. In all cases, the arthroscopy was performed through routine anterolateral and anteromedial portals. None of the patients had a femoral or a sciatic nerve block subsequent to the procedure. No drains were used in any case.

\section{Postoperative management}

The patients underwent the operation on a day-case basis. The same anti-inflammatory and anticoagulant medication was given to all the patients during the postoperative period. All patients passed through a physical therapy program either in our institution or in external rehabilitation facilities after discharge. In both cases, the same postoperative guidelines were respected. It was a standardized physical therapy protocol based on immediate postoperative weight-bearing with crutches as tolerated, without bracing, until there was a normal gait pattern. Range-of-motion was not limited and progressed as tolerated.

The progressive program that the patients followed included strengthening, proprioception and coordination and cardiovascular exercises. The program included targeted strengthening exercises for the lower extremity muscles (quadriceps, hamstrings, hip and calf muscles). It went from isometric exercises to open chain exercises over 6 weeks. For the first 3-4 weeks after surgery, knee flexion during weight-bearing exercises (e.g. squats, lunges) was limited to $60^{\circ}$. Exercise intensity, of a maximum of one repetition, ranged between 65 and $80 \%$ and the volume was three sets of 12-20 repetitions. With a focus on neuromuscular control of the operated knee, proprioception and coordination exercises included moderate intensity tasks (e.g. single-leg balance and static and dynamic stabilization drills on stable and unstable surfaces for $10 \mathrm{~min} / \mathrm{sessions}$ ). The 10-min cardiovascular exercise session called for cycling at light-tomoderate intensity. All patients received the same standardized physical therapy protocol after the surgery.

\section{Outcome assessment}

Patients were assigned to a group depending on how they responded to a question relative to the presence of PFP at the preoperative visit and at 6 weeks after surgery ("Have you ever had pain in the anterior part of the knee in addition to the current pain on the medial or lateral joint line?"). The question was then answered in writing by the patient.

To quantify VM and VL muscle thickness, magnetic resonance imaging (MRI) of the thigh was taken for all patients before surgery and at 6 weeks after surgery. Those MRIs were done on both the injured knee and the contralateral knee. A high correlation coefficient exists between the quadriceps cross-sectional area and the total muscle volume [22]. The knees were imaged on the sagittal plane on the same $1.5 \mathrm{~T}$ whole-body MRI unit (GE SIGNA EXCITE) using a commercial receive-only extremity coil. A topogram was taken and axial planes were programmed in a $\mathrm{T} 1$ fast spin-echo $2 \mathrm{D}$ sequence (flip angle $55^{\circ}$, repetition time $580 \mathrm{~ms}$, minimum TE time $11.30 \mathrm{~ms}$, field of view $17 \times 17 \mathrm{~cm}, 60$ partitions, $448 \times 288$ pixel matrix, acquisition time $2.55 \mathrm{~min}$ ). Sagittal images were obtained at a partition thickness of $6 \mathrm{~mm}$ with a partition interval of $4.50 \mathrm{~mm}$ and an in-plane resolution of $0.31-0.83 \mathrm{~mm}$. All the MRI assessments were blinded to subject identification, time sequences and other knee structural measurements. This measurement was performed at $3.75 \mathrm{~cm}$ for the VM and $15 \mathrm{~cm}$ for the VM and VL from the upper pole of the patella, in accordance with Wang et al. [37]. The VL/VM ratio was calculated with those values [26]. All of the MRI measurements were performed by two blinded radiologists, specialized in musculoskeletal system, as independent observers.

Likewise, the electrical contractility of the quadriceps femoris was analysed with surface electromyography (S-EMG) (MEGAWIN), extracting muscle activity and the maximum voluntary contraction values of the VL and the VM during the preoperative period and at 6 weeks after surgery. Four $\mathrm{Ag} / \mathrm{AgCl}$ surface electrodes $(30 \mathrm{~mm}$ diameter) were distributed in the direction of the muscle fibres of the VM and VL, in accordance with the method for electrode placement in lower limb muscles S-EMG recordings described by Rainoldi et al. [28]. Two additional control electrodes were placed on the bone surface of medial and lateral tibial plateau. A 95\% alcohol solution was used to clean the skin under the electrodes.

Patients were first informed about with electrical stimulation. Then, a single current intensity of 1-ms rectangular pulses was progressively increased in 10-mA steps (starting from $0 \mathrm{~mA}$ ) every 3 to $5 \mathrm{~s}$. Maximal current intensity was determined as the current level at which the evoked torque did not further increase despite increasing current intensity, indicating full quadriceps recruitment. Subsequently, the patients executed a standardized warm-up protocol consisting of 6 submaximal voluntary contractions and 1 maximum voluntary contraction (MVC) with 90 degrees of knee flexion. Next, patients completed 3 MVC trials separated by approximately $30 \mathrm{~s}$. Standardized verbal encouragement and visual feedback were consistently provided to the patients. MVC torque was measured as the peak torque adjusted to body mass attained before or after the superimposed twitch [27]. The activation level was calculated using the following formula: [100 - (superimposed twitch torque/potentiated twitch torque) $\times 100]$. [38]

An isokinetic test (Biodex dynamometer) was also performed both before surgery and 6 weeks after surgery to 
Table 1 Study variables analysed

\begin{tabular}{lccc}
\hline & PFP group $(n=20)$ & Control group $(n=64)$ & $p$ value \\
\hline Preoperative & & & \\
VL $15 \mathrm{~cm}\left(\mathrm{~cm}^{2}\right)$ & $21.1 \pm 3.6$ & $22.3 \pm 3.7$ & n.s \\
VM $15{\mathrm{~cm}\left(\mathrm{~cm}^{2}\right)}^{2}$ & $15.8 \pm 2.9$ & $17.2 \pm 2.7$ & n.s \\
VM 3.75 $\mathrm{cm}^{2}\left(\mathrm{~cm}^{2}\right)$ & $17.7 \pm 2.6$ & $19.2 \pm 4.0$ & n.s \\
VL MA $(\mu \mathrm{V})$ & $2418.3 \pm 940.9$ & $2686.0 \pm 984.8$ & n.s \\
VM MA $(\mu \mathrm{V})$ & $2477.1 \pm 936.3$ & $2626.9 \pm 914.4$ & n.s \\
VL MVC $(\mu \mathrm{V})$ & $266.9 \pm 70.8$ & $264.4 \pm 115.5$ & n.s \\
VM MVC $(\mu \mathrm{V})$ & $271.2 \pm 80.7$ & $248.9 \pm 109.2$ & n.s \\
MS 60 degrees per s $(\mathrm{Kg})$ & $23.6 \pm 8.6$ & $25.1 \pm 9.2$ & n.s \\
Postoperative & & & \\
VL 15 cm $\left(\mathrm{cm}^{2}\right)$ & $15.9 \pm 2.5$ & $20.7 \pm 3.3$ & $<\mathbf{0 . 0 1}$ \\
VM 15 cm $\left(\mathrm{cm}^{2}\right)$ & $9.0 \pm 2.2$ & $14.9 \pm 2.9$ & $<\mathbf{0 . 0 1}$ \\
VM 3.75 cm $\left(\mathrm{cm}^{2}\right)$ & $9.7 \pm 1.8$ & $16.6 \pm 3.9$ & $<\mathbf{0 . 0 1}$ \\
VL MA $(\mu \mathrm{V})$ & $1614.0 \pm 671.7$ & $2199.1 \pm 840.2$ & $\mathbf{0 . 0 2}$ \\
VM MA $(\mu \mathrm{V})$ & $1226.3 \pm 565.8$ & $1946.1 \pm 799.3$ & $<\mathbf{0 . 0 1}$ \\
VL MVC $(\mu \mathrm{V})$ & $159.8 \pm 55.9$ & $222.3 \pm 63.3$ & $\mathbf{0 . 0 4}$ \\
VM MVC $(\mu \mathrm{V})$ & $122.9 \pm 63.9$ & $231.8 \pm 62.8$ & $<\mathbf{0 . 0 1}$ \\
MS 60 degrees per s $(\mathrm{Kg})$ & $12.27 \pm 5.6$ & $20.0 \pm 5.9$ & $<\mathbf{0 . 0 1}$ \\
\hline D & & &
\end{tabular}

Data are reported as mean \pm SD. Bold $p$ values indicate a statistically significant difference between groups $(p<0.05)$. $V L$ vastus lateralis, $V M$ vastus medialis, $V M O$ vastus medialis oblique, $M A$ muscle activity, $M V C$ maximum voluntary contraction, $M S$ muscular strength

assess the muscle performance values. The tests provided data on muscular strength through range of motion at 60 degrees per second. The patients did the extension, with passive return to the starting position. Three repetitions were performed, and the median was chosen for each patient. The electrophysiological and isokinetic tests were performed on both knees by the same physiotherapist who was blinded as to whether the patient had patellofemoral pain.

\section{Statistical analysis}

Numerical variables are expressed descriptively as mean and standard deviations. Within the groups, changes (preoperative vs. postoperative) were evaluated by means of paired $t$ tests. This was performed separately for the PFP group and the control group. The correlation between continuous variables were evaluated with Spearman's rank correlation coefficients. STATA version 15.10 (StataCorp, College Station, TX, USA) was used for the statistical analysis. $p$ values of 0.05 were considered statistically significant.

A sample size calculation was made beforehand. Accepting an alpha risk of 0.05 , a beta risk of 0.20 and a relative risk greater or equal to $0.10,88$ subjects were needed. The proportion of patients who developed PFP after surgery was estimated to be 0.25 , the same as the incidence in healthy people [29, 31, 34]. A follow-up loss of 5\% was assumed. The Poisson approximation was used.

Results
Of the remaining 90 patients after exclusion, 6 were lost to follow-up. The losses included 4 patients from the control group and 2 patients from PFP group. These follow-up losses were found to be non-differential for the statistical analysis of the data, because they did not affect the demographics of the two groups. Of the remaining 84 patients, $20(23.8 \%)$ were allocated to PFP group for developing postoperative anterior knee pain, and 64 patients (76.2\%) were considered controls. The mean age of the sample was 44.9 years (SD 11.0 years). There were 29 women $(34.5 \%)$ and 55 men $(65.5 \%)$. Both groups were comparable in terms of all the preoperative variables analysed (Table 1).

There is a moderate positive correlation between preoperative quadriceps muscle thickness and preoperative muscle activity, MVC and strength values, regardless of whether the patient develops patellofemoral pain or not. This correlation is stronger for the VM at $3.75 \mathrm{~cm}$ of the patella $(0.6$, $p<0.01$ ) (Tables 1, 2, 3, 4). A moderate to high positive correlation exists between preoperative quadriceps femoris muscle thickness and muscle thickness at 6 weeks after surgery. This correlation is independent of whether the patient develops patellofemoral pain or not (Table 2).

There was a moderate positive correlation between preoperative quadriceps femoris muscle thickness and muscle activity, MVC and strength values at 6 weeks after surgery exclusively for the group of patients who do not develop patellofemoral pain. The group of patients who developed patellofemoral pain in the postoperative period did not show this correlation (Table 2, 3, 4). 
Table 2 Postoperative correlations

\begin{tabular}{|c|c|c|c|}
\hline \multirow[t]{2}{*}{ Preoperative } & \multicolumn{3}{|c|}{ Control group $(n=64)$} \\
\hline & VL $15 \mathrm{~cm}$ & VM $15 \mathrm{~cm}$ & VM $3.75 \mathrm{~cm}$ \\
\hline \multicolumn{4}{|l|}{ Preoperative } \\
\hline VL MA & $0.4(\boldsymbol{p}=\mathbf{0 . 0 1})$ & $0.3(\boldsymbol{p}=\mathbf{0 . 0 1})$ & $0.2(\boldsymbol{p}=\mathbf{0 . 0 3})$ \\
\hline VM MA & 0.3 ( $p=$ n.s. $)$ & $0.4(p=\mathbf{0 . 0 8})$ & $0.6(\boldsymbol{p}<\mathbf{0 . 0 1})$ \\
\hline VL MVC & $0.4(\boldsymbol{p}=\mathbf{0 . 0 3})$ & $0.2(p=$ n.s. $)$ & $0.3(\boldsymbol{p}=\mathbf{0 . 0 1})$ \\
\hline VM MVC & $0.2(\boldsymbol{p}=\mathbf{0 . 0 3})$ & $0.4(\boldsymbol{p}=\mathbf{0 . 0 3})$ & $0.4(\boldsymbol{p}<\mathbf{0 . 0 1})$ \\
\hline MS 60 degrees per s & $0.5(p=\mathbf{0 . 0 4})$ & $0.4(\boldsymbol{p}=\mathbf{0 . 0 1})$ & $0.4(\boldsymbol{p}=\mathbf{0 . 0 2})$ \\
\hline \multicolumn{4}{|l|}{ Postoperative } \\
\hline VL $15 \mathrm{~cm}$ & $0.7(\boldsymbol{p}<\mathbf{0 . 0 1})$ & $0.3(\boldsymbol{p}=\mathbf{0 . 0 1})$ & $0.5(\boldsymbol{p}<\mathbf{0 . 0 1})$ \\
\hline VM $15 \mathrm{~cm}$ & $0.2(\boldsymbol{p}=\mathbf{0 . 0 4})$ & $0.8(\boldsymbol{p}<\mathbf{0 . 0 1})$ & $0.4(\boldsymbol{p}<\mathbf{0 . 0 1})$ \\
\hline VM $3.75 \mathrm{~cm}$ & $0.6(p=$ n.s. $)$ & $0.4(\boldsymbol{p}=\mathbf{0 . 0 1})$ & $0.7(\boldsymbol{p}<\mathbf{0 . 0 1})$ \\
\hline VL MA & $0.6(\boldsymbol{p}=\mathbf{0 . 0 4})$ & 0.1 ( $p=$ n.s. $)$ & $0.3(\boldsymbol{p}=\mathbf{0 . 0 3})$ \\
\hline VM MA & 0.3 ( $p=$ n.s. $)$ & $0.7(p=\mathbf{0 . 0 4})$ & $0.5(\boldsymbol{p}=\mathbf{0 . 0 1})$ \\
\hline VL MVC & $0.5(\boldsymbol{p}<\mathbf{0 . 0 1})$ & $0.4(\boldsymbol{p}=\mathbf{0 . 0 2})$ & $0.3(\boldsymbol{p}=\mathbf{0 . 0 4})$ \\
\hline VM MVC & $0.2(\boldsymbol{p}=\mathbf{0 . 0 3})$ & $0.3(p=$ n.s. $)$ & $0.5(\boldsymbol{p}=\mathbf{0 . 0 2})$ \\
\hline MS 60 degrees per s & $0.4(\boldsymbol{p}=\mathbf{0 . 0 2})$ & $0.4(\boldsymbol{p}=\mathbf{0 . 0 1})$ & $0.5(\boldsymbol{p}=\mathbf{0 . 0 1})$ \\
\hline \multirow[t]{2}{*}{ Preoperative } & \multicolumn{3}{|c|}{ PFP group $(n=20)$} \\
\hline & VL $15 \mathrm{~cm}$ & VM $15 \mathrm{~cm}$ & VM $3.75 \mathrm{~cm}$ \\
\hline \multicolumn{4}{|l|}{ Preoperative } \\
\hline VL MA & $0.3(\boldsymbol{p}=\mathbf{0 . 0 4})$ & $0.1(p=$ n.s. $)$ & $0.1(p=$ n.s. $)$ \\
\hline VM MA & $0.3(p=$ n.s. $)$ & $0.3(p=\mathbf{0 . 0 4})$ & $0.4(\boldsymbol{p}=\mathbf{0 . 0 2})$ \\
\hline VL MVC & $0.3(\boldsymbol{p}=\mathbf{0 . 0 4})$ & $0.1(p=\mathrm{n} . \mathrm{s})$. & 0.3 ( $p=$ n.s. $)$ \\
\hline VM MVC & $0.2(p=$ n.s. $)$ & $0.3(p=$ n.s. $)$ & $0.0(p=$ n.s. $)$ \\
\hline MS 60 degrees per s & $0.1(p=\mathrm{n} . \mathrm{s})$. & $0.4(\boldsymbol{p}=\mathbf{0 . 0 3})$ & $0.1(p=$ n.s. $)$ \\
\hline \multicolumn{4}{|l|}{ Postoperative } \\
\hline VL $15 \mathrm{~cm}$ & $0.5(\boldsymbol{p}=\mathbf{0 . 0 2})$ & $0.5(p=\mathbf{0 . 0 4})$ & $0.5(\boldsymbol{p}<\mathbf{0 . 0 1})$ \\
\hline VM $15 \mathrm{~cm}$ & $0.23(\boldsymbol{p}=\mathbf{0 . 0 4})$ & $0.6(\boldsymbol{p}<\mathbf{0 . 0 1})$ & $0.4(\boldsymbol{p}=\mathbf{0 . 0 4})$ \\
\hline VMO or VM $3.75 \mathrm{~cm}$ & $0.4(p=$ n.s. $)$ & $0.6(p=\mathbf{0 . 0 1})$ & $0.6(\boldsymbol{p}<\mathbf{0 . 0 1})$ \\
\hline VL MA & $0.1(p=\mathrm{n} . \mathrm{s})$. & $0.2(p=$ n.s. $)$ & $0.1(p=$ n.s. $)$ \\
\hline VM MA & $0.1(p=$ n.s. $)$ & $0.1(p=$ n.s. $)$ & $0.2(p=$ n.s. $)$ \\
\hline VL MVC & $0.1(p=$ n.s. $)$ & $0.1(p=$ n.s. $)$ & $0.2(p=$ n.s. $)$ \\
\hline VM MVC & 0.1 ( $p=$ n.s. $)$ & 0.1 ( $p=$ n.s. $)$ & 0.1 ( $p=$ n.s. $)$ \\
\hline MS 60 degrees per s & $0.1(p=$ n.s. $)$ & $0.5(p=$ n.s. $)$ & $0.1(p=$ n.s. $)$ \\
\hline
\end{tabular}

Data are reported as mean \pm SD. Bold $p$ values indicate a statistically significant difference between groups $(p<0.05)$. $V L$ vastus lateralis, $V M$ vastus medialis, $V M O$ vastus medialis oblique, $M A$ muscle activity, $M V C$ maximum voluntary contraction, $M S$ muscular strength

\section{Discussion}

The most important finding of the current investigation is that while a positive correlation exists between preoperative quadriceps femoris thickness and postoperative neuromuscular activation in patients without patellofemoral pain, this does not occur in those patients who develop patellofemoral pain after meniscal surgery. The results suggest that the delayed onset of electromyographic activity of the vastus lateralis and especially the vastus medialis muscle, regardless of muscle thickness prior to surgery, could be considered as a risk factor for the development of patellofemoral pain around the sixth week after APM.

It is likely that there are a wide range of factors involved in the aetiology of anterior knee pain [10, 32, 33]. Muscle atrophy as well as the delay in the activation of the quadriceps femoris muscle have already been identified as risk factors for developing patellofemoral pain after arthroscopic knee surgery [1]. Quadriceps femoris muscle thickness has been decreased between 25 and 50\% in the PFP group. Moreover, all the other measurement decreased remarkably compared to the control group. 
Table 3 Postop correlations

\begin{tabular}{llll}
\hline Postoperative & \multicolumn{3}{l}{ Control group $(n=64)$} \\
\cline { 2 - 4 } & VL $15 \mathrm{~cm}$ & VM $15 \mathrm{~cm}$ & VM $3.75 \mathrm{~cm}$ \\
\hline Postoperative & & & \\
VL MA & $0.5(\boldsymbol{p}=\mathbf{0 . 0 1})$ & $0.2(p=$ n.s. $)$ & $0.3(\boldsymbol{p}=\mathbf{0 . 0 3})$ \\
VM MA & $0.2(\boldsymbol{p}=\mathbf{0 . 0 3})$ & $0.5(\boldsymbol{p}=\mathbf{0 . 0 4})$ & $0.5(\boldsymbol{p}=\mathbf{0 . 0 4})$ \\
VL MVC & $0.4(\boldsymbol{p}<\mathbf{0 . 0 1})$ & $0.2(\boldsymbol{p}<\mathbf{0 . 0 1})$ & $0.2(\boldsymbol{p}<\mathbf{0 . 0 1})$ \\
VM MVC & $0.2(p=$ n.s. $)$ & $0.3(p=$ n.s. $)$ & $0.5(\boldsymbol{p}=\mathbf{0 . 0 4})$ \\
$\quad \begin{array}{l}\text { MS 60 degrees } \\
\text { per s }\end{array}$ & $0.3(\boldsymbol{p}=\mathbf{0 . 0 2})$ & $0.4(\boldsymbol{p}=\mathbf{0 . 0 1})$ & $0.5(\boldsymbol{p}=\mathbf{0 . 0 3})$ \\
\hline
\end{tabular}

Data are reported as mean \pm SD. Bold $p$ values indicate a statistically significant difference between groups $(p<0.05)$. $V L$ vastus lateralis, $V M$ vastus medialis, $V M O$ vastus medialis oblique, $M A$ muscle activity, $M V C$ maximum voluntary contraction, $M S$ muscular strength

Table 4 Postop correlations

\begin{tabular}{llll}
\hline Postoperative & \multicolumn{2}{l}{ PFP group $(n=20)$} \\
\cline { 2 - 4 } & VL 15 cm & VM $15 \mathrm{~cm}$ & VM 3.75 cm \\
\hline Postoperative & $0.1(p=$ n.s. $)$ & $0.3(p=$ n.s. $)$ & $0.3(p=$ n.s. $)$ \\
VL MA & $0.1(p=$ n.s. $)$ & $0.2(p=$ n.s. $)$ & $0.1(p=$ n.s. $)$ \\
VM MA & $0.1(p=$ n.s. $)$ & $0.2(p=$ n.s. $)$ & $0.2(p=$ n.s. $)$ \\
VL MVC & $0.0(p=$ n.s. $)$ & $0.1(p=$ n.s. $)$ & $0.2(p=$ n.s. $)$ \\
VM MVC & $0.2(p<\mathbf{0 . 0 1})$ & $0.1(p=$ n.s. $)$ & $0.3(p=$ n.s. $)$ \\
\hline MS 60 degrees per s &
\end{tabular}

Data are reported as mean $\pm \mathrm{SD}$. Bold $p$ values indicate a statistically significant difference between groups $(p<0.05)$. $V L$ vastus lateralis, $V M$ vastus medialis, $V M O$ vastus medialis oblique, $M A$ muscle activity, $M V C$ maximum voluntary contraction, $M S$ muscular strength

The results indicate that patients in whom PFP appears after arthroscopic surgery experience muscular atrophy of the VL and, to a greater extent, the VM during a period of 6 weeks. This decrease in quadriceps femoris muscle size is probably related to postoperative proximity inhibition and the consequent failure of muscle activation. Then again, it might also be related to the development of PFP. This article goes further, as it shows the association between quadriceps activation failure and postoperative patellofemoral pain, even in those patients with good muscle thickness and good electrical contractility of the quadriceps muscles prior to surgery. In this line, QAF and QMH are crucial factors to target to improve the recovery of knee function following knee arthroscopy.

Another interesting finding of the current work is the incidence of PFP after an arthroscopic meniscectomy in patients who did not previously have this pain. The $23.8 \%$ incidence of postoperative PFP is similar to that of patients after ACL reconstruction at the 1- and 2-year follow-up (24\% and 22\%, respectively) [5]. However, the shorter follow-up time in the present investigation impedes drawing any firm conclusion with regard to this particular issue.

Recently, research has focused on developing specific disinhibitory interventions to improve voluntary quadriceps activation. Neuromuscular electrical stimulation has been shown to improve quadriceps function and strength, as well as decrease its atrophy in the postoperative period of ACL surgery $[15,19]$. Eccentric exercise, whereby the muscle is lengthened and an external force exceeds that produced by the muscle, has been shown to be more effective than traditional concentric strengthening at minimizing muscle atrophy and improving muscle force production [9]. The ability to eccentrically contract the quadriceps is critical for optimal knee range of motion during the weight-acceptance phase of gait $[11,36]$, which is necessary in the early phase of rehabilitation after meniscal surgery $[2,3,16,18]$. The combination of neuromuscular electrical stimulation with eccentric exercises in the postoperative rehabilitation protocol after meniscal surgery may improve early activation of the quadriceps femoris muscle. Therefore, they may aid in preventing the development of anterior knee pain, even in those patients with poor quadriceps muscle thickness.

A threshold of 6 weeks was set for the measurements. It is the moment in which the incidence of patellofemoral pain increases after knee arthroscopy [1, 2]. In general terms, although the treatment must be individualized for each patient, sixth weeks is the time point from which the patient should be able to return to play after an APM [16]. It is at this point that the patient should be able to fully activate the quadriceps femoris muscle.

A progressive pre-habilitation program that is mainly focused on strengthening the quadriceps femoris of subjects who have undergone meniscal surgery leads to improved knee function in the postoperative period, in the same way as happens in patients who have undergone ACL reconstruction $[6,8,15,19,24]$. However, based on the current results, those patients who develop patellofemoral pain after arthroscopy do not show this correlation.

There are some limitations in the present study. One is the severity of meniscal damage and consequently the amount of meniscus removed at surgery, as they might have an impact on the degree of postoperative electrical contractility of the quadriceps femoris. Then, the definition of PFP, which is based on the presence of pain in the anterior part of the knee in a self-referral manner and not on more objective and specific measure score or patellofemoral questionnaires like the patellar diagnostic test (Felson). [35] Lower extremity structural anomalies on the transverse plane like increased femoral anteversion and lateral tibial torsion may contribute to patellofemoral malalignment and PFP must also be considered [4, 7]. These factors have not been analysed in the current study. Thus, that might be another limitation. 
In the light of the current results, early activation of the quadriceps femoris after APM is particularly important for the prevention of postoperative patellofemoral pain, regardless of the quadriceps muscle thickness prior to the intervention.

This study provide insights on the influence of neuromuscular control on anterior knee pain and how the postoperative rehabilitation protocol after meniscectomy should be approach. Those results may in turn be useful in guiding rehabilitation efforts and guide daily clinical practice after knee arthroscopy.

\section{Conclusion}

In patients without patellofemoral pain after meniscectomy, the thicker the preoperative quadriceps femoris, the more postoperative neuromuscular activation and strength they have. This correlation did not occur in those patients who develop patellofemoral pain after meniscal surgery.

Acknowledgements The authors thank the Radiology Department of Hospital del Mar for their help in performing the measurements on the MR images. They also thank Mr. Sanchez and Ms. Piqueras, physiotherapists from the INVALCOR Biomechanical Center, who collaborated in carrying out all of the isokinetic and electromyographic tests on our patients. This study was done within the framework of the $\mathrm{PhD}$ program of the Department of Surgery and Morphological Sciences of the Universitat Autònoma de Barcelona (UAB).

Funding Open Access Funding provided by Universitat Autonoma de Barcelona.

\section{Declarations}

Conflict of interest The authors declare that they have no conflict of interest.

Ethical approval Approval for the study was granted by the Ethics Committee of Clinical Research of Parc de Salut Mar Hospital, Autonomous University of Barcelona (CEIC no. 2014/5534)

Open Access This article is licensed under a Creative Commons Attribution 4.0 International License, which permits use, sharing, adaptation, distribution and reproduction in any medium or format, as long as you give appropriate credit to the original author(s) and the source, provide a link to the Creative Commons licence, and indicate if changes were made. The images or other third party material in this article are included in the article's Creative Commons licence, unless indicated otherwise in a credit line to the material. If material is not included in the article's Creative Commons licence and your intended use is not permitted by statutory regulation or exceeds the permitted use, you will need to obtain permission directly from the copyright holder. To view a copy of this licence, visit http://creativecommons.org/licenses/by/4.0/.

\section{References}

1. Amestoy J, Pérez-Prieto D, Torres-Claramunt R, Sánchez-Soler JF, Leal-Blanquet J, Ares-Vidal J, Hinarejos P, Monllau JC (2021) Patellofemoral pain after arthroscopy: muscle atrophy is not everything. Orthop J Sports Med 9:232596712110130

2. Becker R, Kopf S, Seil R, Hirschmann MT, Beaufils P, Karlsson J (2020) From meniscal resection to meniscal repair: a journey of the last decade. Knee Surg Sports Traumatol Arthrosc 28:3401-3404

3. Capin JJ, Khandha A, Zarzycki R, Manal K, Buchanan TS, Snyder-Mackler L (2018) Gait mechanics after ACL reconstruction differ according to medial meniscal treatment. J Bone Joint Surg Am 100:1209-1216

4. Cibulka MT, Threlkeld-Watkins J (2005) Patellofemoral pain and asymmetrical hip rotation. Phys Ther 85:1201-1207

5. Culvenor AG, Øiestad BE, Holm I, Gunderson RB, Crossley KM, Risberg MA (2017) Anterior knee pain following anterior cruciate ligament reconstruction does not increase the risk of patellofemoral osteoarthritis at 15- and 20-year follow-ups. Osteoarthr Cartil 25:30-33

6. Eitzen I, Holm I, Risberg MA (2009) Preoperative quadriceps strength is a significant predictor of knee function two years after anterior cruciate ligament reconstruction. Br J Sports Med 43:371-376

7. Erkocak OF, Altan E, Altintas M, Turkmen F, Aydin BK, Bayar A (2016) Lower extremity rotational deformities and patellofemoral alignment parameters in patients with anterior knee pain. Knee Surg Sports Traumatol Arthrosc 24:3011-3020

8. Failla MJ, Arundale AJH, Logerstedt DS, Snyder-Mackler L (2015) Controversies in knee rehabilitation. Clin Sports Med 34:301-312

9. Gerber JP, Marcus RL, Dibble LE, Greis PE, Burks RT, LaStayo PC (2007) Effects of early progressive eccentric exercise on muscle structure after anterior cruciate ligament reconstruction. J Bone Joint Surg Am 89:559-570

10. Goicoechea N, Hinarejos P, Torres-Claramunt R, Leal-Blanquet J, Sánchez-Soler J, Monllau JC (2021) Patellar denervation does not reduce post-operative anterior knee pain after primary total knee arthroplasty with patellar resurfacing. Knee Surg Sports Traumatol Arthrosc 29:3346-3351

11. Harkey MS, Gribble PA, Pietrosimone BG (2014) Disinhibitory interventions and voluntary quadriceps activation: a systematic review. J Athl Train 49:411-421

12. Hart JM, Pietrosimone B, Hertel J, Ingersoll CD (2010) Quadriceps activation following knee injuries: a systematic review. J Athl Train 45:87-97

13. Hurley MV, Jones DW, Newham DJ (1994) Arthrogenic quadriceps inhibition and rehabilitation of patients with extensive traumatic knee injuries. Clin Sci (Lond) 86:305-310

14. Ingersoll CD, Grindstaff TL, Pietrosimone BG, Hart JM (2008) Neuromuscular consequences of anterior cruciate ligament injury. Clin Sports Med 27(383-404):vii

15. Johnston PT, Feller JA, McClelland JA, Webster KE (2021) Knee strength deficits following anterior cruciate ligament reconstruction differ between quadriceps and hamstring tendon autografts. Knee Surg Sports Traumatol Arthrosc. https://doi.org/10.1007/ s00167-021-06565-0

16. Kopf S, Beaufils P, Hirschmann MT, Rotigliano N, Ollivier M, Pereira H, Verdonk R, Darabos N, Ntagiopoulos P, Dejour D, Seil R, Becker R (2020) Management of traumatic meniscus tears: the 2019 ESSKA meniscus consensus. Knee Surg Sports Traumatol Arthrosc 28:1177-1194 
17. Krishnan C, Williams GN (2011) Factors explaining chronic knee extensor strength deficits after ACL reconstruction. J Orthop Res 29:633-640

18. Lepley LK, Lepley AS, Onate JA, Grooms DR (2017) Eccentric exercise to enhance neuromuscular control. Sports Health 9:333-340

19. Lepley LK, Wojtys EM, Palmieri-Smith RM (2015) Combination of eccentric exercise and neuromuscular electrical stimulation to improve quadriceps function post-ACL reconstruction. Knee 22:270-277

20. Lopresti C, Kirkendall DT, Street GM, Dudley AW (1988) Quadriceps insufficiency following repair of the anterior cruciate ligament. J Orthop Sports Phys Ther 9:245-249

21. Lorentzon R, Elmqvist LG, Sjöström M, Fagerlund M, Fuglmeyer AR (1989) Thigh musculature in relation to chronic anterior cruciate ligament tear: muscle size, morphology, and mechanical output before reconstruction. Am J Sports Med 17:423-429

22. Marcon M, Ciritsis B, Laux C, Nanz D, Nguyen-Kim TDL, Fischer MA, Andreisek G, Ulbrich EJ (2015) Cross-sectional area measurements versus volumetric assessment of the quadriceps femoris muscle in patients with anterior cruciate ligament reconstructions. Eur Radiol 25:290-298

23. Narici M, Cerretelli P (1998) Changes in human muscle architecture in disuse-atrophy evaluated by ultrasound imaging. J Gravit Physiol 5:P73-74

24. Palmieri-Smith RM, Lepley LK (2015) Quadriceps strength asymmetry after anterior cruciate ligament reconstruction alters knee joint biomechanics and functional performance at time of return to activity. Am J Sports Med 43:1662-1669

25. Palmieri-Smith RM, Thomas AC, Wojtys EM (2008) Maximizing quadriceps strength after ACL reconstruction. Clin Sports Med 27:405-424

26. Pan J, Stehling C, Muller-Hocker C, Schwaiger BJ, Lynch J, McCulloch CE, Nevitt MC, Link TM (2011) Vastus lateralis/ vastus medialis cross-sectional area ratio impacts presence and degree of knee joint abnormalities and cartilage T2 determined with 3T MRI - an analysis from the incidence cohort of the Osteoarthritis Initiative. Osteoarthr Cartil 19:65-73

27. Place N, Maffiuletti NA, Martin A, Lepers R (2007) Assessment of the reliability of central and peripheral fatigue after sustained maximal voluntary contraction of the quadriceps muscle. Muscle Nerve 35:486-495

28. Rainoldi A, Melchiorri G, Caruso I (2004) A method for positioning electrodes during surface EMG recordings in lower limb muscles. J Neurosci Methods 134:37-43
29. Rathleff MS, Vicenzino B, Middelkoop M, Graven-Nielsen T, van Linschoten R, Hölmich P, Thorborg K (2015) Patellofemoral pain in adolescence and adulthood: same, but different? Sports Med 45:1489-1495

30. Rice DA, McNair PJ (2010) Quadriceps arthrogenic muscle inhibition: neural mechanisms and treatment perspectives. Semin Arthritis Rheum 40:250-266

31. Roush JR, Bay RC (2012) Prevalence of anterior knee pain in 18-35 year-old females. Int J Sports Phys Ther 7:396-401

32. Sanchis-Alfonso V (2008) Patellofemoral pain. Orthopade 37(835-836):838-840

33. Sanchis-Alfonso V (2014) Holistic approach to understanding anterior knee pain. Clinical implications. Knee Surg Sports Traumatol Arthrosc 22:2275-2285

34. Smith BE, Selfe J, Thacker D, Hendrick P, Bateman M, Moffatt F, Rathleff MS, Smith TO, Logan P (2018) Incidence and prevalence of patellofemoral pain: a systematic review and meta-analysis. PLoS ONE 13:e190892

35. Stefanik JJ, Neogi T, Niu J, Roemer FW, Segal NA, Lewis CE, Nevitt M, Guermazi A, Felson DT (2014) The diagnostic performance of anterior knee pain and activity-related pain in identifying knees with structural damage in the patellofemoral joint: the Multicenter Osteoarthritis Study. J Rheumatol 41:1695-1702

36. Torry MR, Decker MJ, Viola RW, O'Connor DD, Steadman JR (2000) Intra-articular knee joint effusion induces quadriceps avoidance gait patterns. Clin Biomech (Bristol, Avon) 15:147-159

37. Wang Y, Wluka AE, Berry PA, Siew T, Teichtahl AJ, Urquhart DM, Lloyd DG, Jones G, Cicuttini FM (2012) Increase in vastus medialis cross-sectional area is associated with reduced pain, cartilage loss, and joint replacement risk in knee osteoarthritis. Arthritis Rheum 64:3917-3925

38. Wellauer V, Morf C, Minetto MA, Place N, Maffiuletti NA (2015) Assessment of quadriceps muscle inactivation with a new electrical stimulation paradigm: quadriceps neuromuscular testing. Muscle Nerve 51:117-124

39. Williams GN, Buchanan TS, Barrance PJ, Axe MJ, Snyder-Mackler L (2005) Quadriceps weakness, atrophy, and activation failure in predicted noncopers after anterior cruciate ligament injury. Am J Sports Med 33:402-407

Publisher's Note Springer Nature remains neutral with regard to jurisdictional claims in published maps and institutional affiliations. 